\title{
Elevated risk for squamous cell carcinoma of the conjunctiva among adults with AIDS in the United States M Guech-Ongey*1, EA Engels ${ }^{1}$, JJ Goedert ${ }^{1}$, RJ Biggar ${ }^{1,2}$ and SM Mbulaiteye ${ }^{1}$
}

Address: ${ }^{1}$ Viral Epidemiology Branch, Division of Cancer Epidemiology and Genetics, National Cancer Institute, Bethesda, Maryland, USA and 2Department of Epidemiology Research, State Serum Institute, Copenhagen, Denmark

* Corresponding author

from I I th International Conference on Malignancies in AIDS and Other Acquired Immunodeficiencies (ICMAOI): Basic, Epidemiologic, and Clinical Research

Bethesda, MD, USA. 6-7 October 2008

Published: 17 June 2009

Infectious Agents and Cancer 2009, 4(Suppl 2):P2 I doi:I0.|I86/I750-9378-4-S2-P2 I

This abstract is available from: http://www.infectagentscancer.com/content/4/S2/P2 I

(c) 2009 Guech-Ongey et al; licensee BioMed Central Ltd.

Squamous cell carcinoma of the conjunctiva (SCCC) has been associated with HIV infection in equatorial Africa, but the evidence for association with HIV in developed countries, where SCCC is rarer, is controversial. We investigated the risk for SCCC and other eye cancers in the updated U.S. HIV/AIDS Cancer Match Registry Study. We calculated standardized incidence ratios (SIRs) to estimate excess risk for SCCC, primary ocular lymphoma, ocular Kaposi sarcoma (KS), and other eye tumors among 491, 048 adults (age $>15$ years or older) with HIV/AIDS diagnosed from 1980 to 2004 . We calculated relative proportions (per $10^{5}$ ) to gain insight into risk factors. We identified 73 eye cancers (15 SCCC, 35 primary ocular lymphoma, 17 ocular KS, and 6 other). Overall SIRs were elevated for SCCC (SIR, 12.2, 95\% CI 6.8 - 20.2), primary ocular lymphoma $(21.7,95 \%$ CI 15.1-30.2), and ocular KS (109, 95\% CI 63.5-175). Risk for SCCC was elevated regardless of HIV acquisition category, CD4 lymphocyte count, and time-relative to AIDS-onset. Relative proportions of SCCC risk were highest with age $\geq 50\left(8 / 10^{5}\right)$, Hispanic ethnicity $\left(7 / 10^{5}\right)$, and residence in regions with high solar ultraviolet radiation $\left(10 / 10^{5}\right)$. We show significantly increased incidence of SCCC among persons with HIV/AIDS in the United States. The associations with age and geography are in accord with etiological role for ultraviolet radiation in SCCC. 